\title{
Phenotypic divergence and inter-specific trait correlation in a plant-pollinator/seed predator mutualism
}

\author{
Isabel S. Magalhaes • Giorgina Bernasconi
}

Received: 16 January 2014/ Accepted: 12 May 2014/Published online: 18 May 2014

(C) Springer International Publishing Switzerland 2014

\begin{abstract}
Plant-pollinator interactions have been suggested as key drivers of morphological divergence and speciation of the involved taxa. These interactions can also promote sexual dimorphism in both the plant and pollinator, particularly if the pollinator is also a seed-eater and/or exerts different selection pressures on male and female plants. Here we tested the hypotheses that plant-pollinator interactions can be reflected in trait variation and sexual dimorphism in both organisms within and across populations. Across nine European populations, we examined intraspecific variation and sexual dimorphism in phenotypic traits potentially involved in the plant-insect interaction of the dioecious white campion Silene latifolia (Caryophyllaceae) and its specialist pollinator Hadena bicruris (Noctuidae). This interaction is expected to entail sex-specific selective pressures, as female moths lay eggs on female plants and the larvae predate on the seeds during their development. We compared divergence in phenotypic traits among populations and between sexes within populations, examined correlations between plant and pollinator traits, and between phenotypic distances and genetic distances among co-occurring populations for both plants and insects. We found key differences in phenotypic traits across populations of both the plant and moth, though only in the moth were these differences correlated with geographic distances. We also found evidence for sexual dimorphism in the plant but not in the pollinator. Evolution of floral sexual dimorphism in $S$. latifolia most likely results from the joint contribution of different selective forces, including biotic interactions with $H$. bicruris moths.
\end{abstract}

Electronic supplementary material The online version of this article (doi:10.1007/s10682-014-9713-z) contains supplementary material, which is available to authorized users.

Present Address:

I. S. Magalhaes

School of Life Sciences, University of Nottingham, University Park, Nottingham NG7 2RD, UK

e-mail: isabel.santos@nottingham.ac.uk

I. S. Magalhaes · G. Bernasconi $(\bowtie)$

Evolutionary Botany, Institute of Biology, University of Neuchâtel, Rue Emile-Argand 11,

2000 Neuchâtel, Switzerland

e-mail: bernasconi.giorgina@gmail.com 
Keywords Parasitism · Pollination · Mutualism · Sexual dimorphism ·

Species interaction

\section{Introduction}

Species interactions can generate benefits and costs that fuel unilateral or reciprocal evolution driven by natural selection. The impact on trait evolution in turn will result in traits that contribute to regulate the interaction itself, raising interest for identifying the involved traits, their effects on costs and benefits of the interaction, and their covariation between interacting species. The directionality and degree of trait covariation (Anderson et al. 2010) will depend on several factors including patterns of gene flow, geographic variation in community structure (e.g., availability of co-pollinators, Thompson 2005) and in environmental conditions (Avilés et al. 2012), but also the degree of specialization in the interaction (see Dufaÿ and Anstett 2003; Kephart et al. 2006), and the plant breeding and mating system (e.g., the extent of reliance on pollinators).

Plant-pollinator mutualisms, which play an important role in survival and reproductive success of both the plant and pollinator populations, are not free of costs. In insectpollinated plants, flowering plants can face a complex dilemma as they must attract pollinators but avoid enemies, whereby these two antagonistic roles can happen at different life stages of the same species. Nursery pollination systems are prime examples of this dilemma as adults act as pollinators and reproduce within the flower or inflorescence they pollinate and their larvae feed on the developing seeds or on other parts of the plant. As a consequence of this, plant morphology is expected to be subjected to selection for both efficient pollination and predation avoidance. Among the best known examples of pollinating seed-consuming systems are the obligate interactions of fig/fig wasp (Anstett et al. 1997; Cook and Rasplus 2003) and yucca/yucca moth (Pellmyr 2003) systems, but at least 13 distinct systems with obligate and non-obligate interactions have been described to date (reviewed by Dufaÿ and Anstett 2003).

In the case of pollinator/seed predator systems that involve dioecious plants, pollinators tend to oviposit on only one of the sexes. In many dioecious systems, the larvae develop in male flowers. In these cases, as male flowers do not produce seeds and generally are infested after having fulfilled their reproductive function, the cost for the plant should be low (Dufaÿ and Anstett 2003). However, in other cases (Kephart et al. 2006), such as in the strong, but non-obligate interactions between plants in the genus Silene and noctuid moths in the genus Hadena (e.g. S. stellate and H. ectypa, Castillo et al. 2013; Kula et al. 2013; or the white campion S. latifolia and its pollinating seed-predator, H. bicruris, Bernasconi et al. 2009) the pollinator larvae develop within the female flower and consume the developing seeds of one or several fruits on the same plant. Thus, in dioecious plants such as $S$. latifolia, seed predation can have a direct impact on female fitness but only an indirect effect on male fitness, leading to the different sexes being subjected to different selective pressures. In the $\mathrm{S}$. latifolia/H. bicruris system, pollen is deposited on the pollinator during feeding (Labouche and Bernasconi 2010), thus male morphological development should favour pollinator attraction and efficient pollen dispersal. For female flowers, oviposition does not increase pollination efficiency compared to nectar feeding alone (Labouche and Bernasconi 2010), but attracting pollinators increases the risk of egg infestation (Elzinga and Bernasconi 2009; Burkhardt et al. 2012). Floral tube morphology may influence 
pollinator access to nectar at the base of the petals (Dötterl and Jürgens 2005; Golonka and Vilgalys 2013) and to the stigmatic surfaces that are receptive for pollen (Burkhardt et al. 2009), but also predicts egg placement, which can in turn influence egg success and the costs of larval infestation (Labouche and Bernasconi 2013). Hence, this and other components of female floral morphology may have evolved not only in response to the pollinator feeding behaviour and pollen deposition, but also to regulate egg fate, and possibly also oviposition by the pollinator, which may be key to reducing plant reproductive costs and thus to the evolutionary stability of these systems (Holland et al. 2004; Labouche and Bernasconi 2013). Sexual dimorphism has been documented for many traits in S. latifolia (Delph and Meagher 1995; Delph et al. 2002, 2010; Delph 2007; Delph and Bell 2008; Yu et al. 2011). Yet, measures of $H$. bicruris phenotypic traits and analyses of how traits potentially involved in the plant-pollinator interaction are correlated have so far been lacking. A combined analysis of traits of plant and pollinator is an essential step to understand trait evolution arising from the species interactions.

In this study, we examined phenotypic variation and correlation in nine populations of the white-campion $S$. latifolia and of the moth $H$. bircruris. We examined the geographic variation across populations in five plant traits and two moth traits likely to be involved in pollination, nectar feeding or oviposition. As we investigated variation in field populations, our study focuses on the analysis of environmental and genetic variation combined. Specifically we addressed three aspects. First, we examined sexual dimorphism in plants and pollinators by comparing within each population the differences in traits between female and male $S$. latifolia and $H$. bicruris. Second, we examined the signature of divergent selection on phenotypic traits by correlating phenotypic divergence and genetic divergence among populations intra-specifically for both plants and moths. Finding significant associations in the degree of among population divergence at both the phenotypic and genetic levels would suggest a potential for local adaptation generating the observed phenotypic differences. Third, we examined phenotypic correlations between species, i.e. between traits of the plants and their pollinators. Finding significant correlations between traits involved in species' interactions will not be sufficient to directly conclude that there is co-evolution of phenotypic traits (Janzen 1980; Nuismer et al. 2010), but it would establish the extent of an association between traits in a set of interacting populations, which could then be used in further studies trying to disentangle the causes for such a correlation.

\section{Materials and methods}

\section{Study system}

Silene latifolia is a dioecious, short-lived perennial plant native to Europe (Delph and Meagher 1995; Shykoff and Bucheli 1995; Witt et al. 1999). Natural patches are often small and isolated (Richards et al. 2003; Elzinga et al. 2005) and occur in disturbed habitats (Goulson and Jerrim 1997). The seed predating moth H. bicruris is considered the main pollinator in the native range, being reported in $90 \%$ of European S. latifolia populations surveyed (Wolfe 2002). However, other moth species occur in S. latifolia populations (e.g. see Magalhaes et al. 2011, Supplementary Information for a list of over thirty Noctuid species collected within three of our study populations) and may act as copollinators. Both male and female $H$. bicruris moths are efficient pollinators (Labouche and Bernasconi 2010). Female adults of this moth oviposit in S. latifolia female flowers 
which they appear to prefer over other host plants in the genus Silene (Pettersson 1991; Bopp and Gottsberger 2004). Once the egg has hatched, the larva enters the fruit (primary fruit), consumes the developing seeds, then leaves and then starts feeding on other (secondary) fruits on the same plant. In primary fruits, if the larva develops successfully, all seeds are destroyed, or do not contribute to seed production because the fruit is aborted (Jolivet and Bernasconi 2006). The moths represent an important cost to the plant as attack rates of $25-70 \%$ of the fruits are common within natural plant populations (Elzinga et al. 2005). Plants respond to experimental (Jolivet and Bernasconi 2006) and natural infestation with increased fruit abortion, with abortion rates varying greatly among populations (Elzinga and Bernasconi 2009).

Silene latifolia plants are also affected by the anther-smut fungus Microbotryum violaceum, a pathogen of the Caryophyllaceae (Thrall et al. 1993). This heterobasidiomycete fungus has the dynamics of a sexually transmitted disease that sterilizes the plant (Alexander and Antonovics 1988; Antonovics and Alexander 1992). Pollinators and other visiting insects serve as vectors of the fungal teliospores (Jennersten 1983; Alexander 1989; Roche et al. 1995). Since the interaction with the fungus is not at the focus of the present study, we have included in our sampling as described below, only plants with normal flowers (i.e. not presenting signs of fungal infestation).

Plant and insect collection and measurement

We sampled flowers and moths from nine geographically separated S. latifolia populations along a north-south transect extending over $1,000 \mathrm{~km}$ (as the crow flies) from southern Sweden to southern Germany: Sörby (from here on S), Jönköping (J), Aarhus (A), Norra Åsum (NA), Gärds Köpinge (GK), Kåseberga (K), Renkum (R), Tolkamer (T) and Stechendorf (ST) (see Supporting Information Figure S1 for map and GPS coordinates of each population). This transect was chosen so as to sample a maximum divergence along the south-north gradient, thus capturing latitude effects, while avoiding known differences between the eastern and western S. latifolia races (Hathaway et al. 2009). Each population was sampled simultaneously for plants and insects (see below) from August 10th, 2007 to September 4th, 2007 whereby R, T and ST were resampled a few weeks after the first field survey to increase moth sample size.

In each population, we sampled 15-20 female and 8-20 male flowering plants (see Table S1 for details on sample sizes per population and sex). In the populations in which we observed plants presenting flowers infested with the anther-smut fungus Microbotryum violaceum (Jönköping, Norra Åsum, Kåseberga, Renkum, Tolkamer and Stechendorf), we included in our samples only plants without signs of fungal infection (i.e. not presenting spores on their flowers), from which we collected leaves for genetic analysis (Magalhaes et al. 2011) and obtained trait measurements (present study, see below). To collect larvae of $\mathrm{H}$. bicruris all flowering female plants (or a subset in the large population A) were inspected by examining developing fruits for holes with protruding excrement (primarily attacked fruits) and ripe fruits for visible larvae (secondarily attacked fruits, Biere 2002). The number of larvae collected ranged from 110 larvae (in population A) to less than 3 larvae (in K and ST, interestingly $M$. violaceum was highly prevalent in these populations). Only in the populations R and T where we could not find any larvae, we collected ca. 10 eggs per population and reared them to adulthood. Thus, age and stage at collection varied, but traits were all measured on adult moths. We kept the eggs in Petri dishes on wet absorbent cotton until hatching; young larvae were transferred individually to plastic vials and fed on artificial diet until pupation (Elzinga et al. 2002). Pupae were stored at $4{ }^{\circ} \mathrm{C}$ 
over winter and then transferred to a growth chamber (SANYO MLR-350H; $25-22{ }^{\circ} \mathrm{C}$, 16 h:8 h day: night, $55 \% \mathrm{RH})$ until emergence of adult moths. During field collections, we stored pupae in single large Petri dishes at $4{ }^{\circ} \mathrm{C}$ in a compressor cooler, EZC 25 12/24 Volt, Ezetil $^{\circledR}$, EZetil E.Zorn GmbH \& Co Vertriebs KG, Hungen-Inheiden, Germany). Mortality between larval stage and pupa was high and not all pupae hatched, so the final sample size of emerged adult moths varied from 7 to 23 moths per population (see Table $\mathrm{S} 1$ for details on sample sizes per population and sex). All of the emerged adults were classified by a specialist taxonomist (Ladislaus Reser, Natural History Museum, Lucerne, Switzerland) and confirmed as belonging to $H$. bicruris.

We identified and measured a number of traits for both the plant and moth that we assumed likely to play a role in the interaction during either pollination or seed predation (Fig. 1). Specifically, we measured (1) corolla tube width (CTW), corolla tube length (CTL; measured only up to the ovary, see Fig. 1; total corolla tube length can thus be estimated as CTL + OvaL) and petal limb length (PL) for both sexes of S. latifolia, and ovary length (OvaL) for females of S. latifolia; (2) proboscis length (PrL) and ovipositor length (OviL) for females of H. bicruris (Fig. 1, also see Labouche and Bernasconi 2013 for details on how measurements were taken). We additionally measured as a control for moth size thorax width (ThW) and wing area (WingA). Wing area was measured automatically in imageJ based on contrast.

Phenotypic assignment of individuals to clusters

We used the model-based clustering method implemented in the package Mclust v4 (Fraley et al. 2012) for R (www.r-project.org) to determine the number of phenotypic clusters among geographically isolated populations based on CTW, CTL and PL pooled together for S. latifolia, and based on PrL, ThW and WingA pooled together for H. bicruris. This method fits the observed frequency distribution of morphological measurements to a series of six distribution models incorporating only one or mixtures of two to six Gaussian distributions and a number of possible clusters within each model, varying from one to nine. The estimated number of clusters is that maximizes the Bayesian Information Criteria (BIC). The higher the BIC, the lower the global average and median classification uncertainty and the better model fits with the data. Once the optimal number of clusters was found we obtained the posterior probability of each individual belonging to each cluster. Sexual dimorphism may mask phenotypic differences among populations, so male and females were non-randomly assigned to two different clusters, suggesting sexual dimorphism, and the analyses were repeated for males and females separately. Phenotypic variation among populations was visualized using boxplots for all analysed traits of $S$. latifolia and of $H$. bicruris separately.

Phenotypic variation and divergence between populations and sexes

We performed MANOVAs on plant and moth traits to test for effects of population, sex and their interaction. All effects were considered fixed. MANOVAs were evaluated using the Pillai's trace test criterion (Scheiner and Gurevitch 2001). Following significant population, sex, or sex*population interactions, we conducted protected ANOVAs (Scheiner and Gurevitch 2001) (type II SS) for each trait, again examining the factors population, sex and their interaction. If the interaction term sex*population was significant we ran one-way ANOVAs combining sex and population into one categorical variable, to determine the populations which exhibited a significant difference between sexes within populations 
(a)

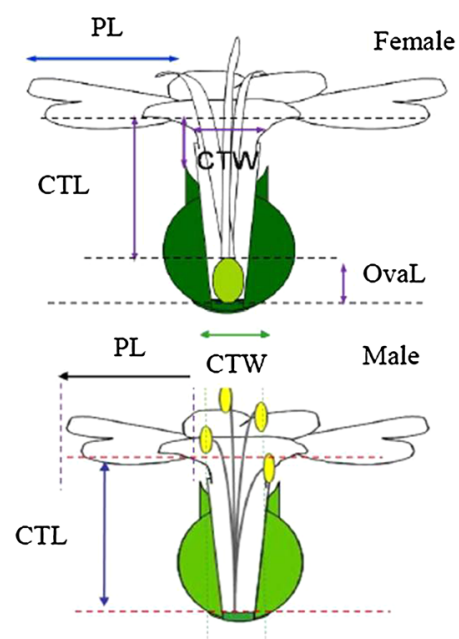

(b)

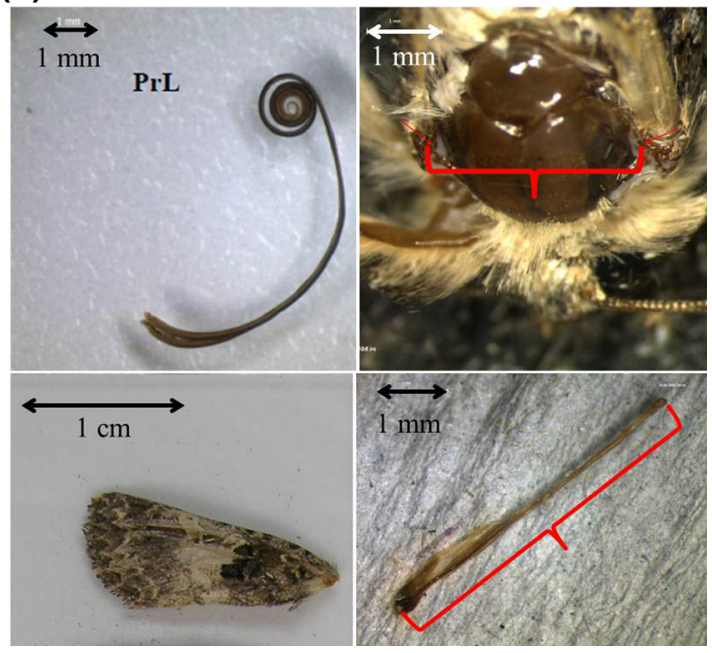

Fig. 1 Morphological traits measured in a Silene latifolia: corolla tube width CTW, corolla tube length CTL, petal length PL and ovary length OvaL; and b Hadena bicruris: proboscis length PrL, wing area WingA, thorax width ThW and ovipositor length OviL. See "Methods" for detailed measurement procedures. Nectar occurs at the base of the petals (Dötterl and Jürgens 2005; Golonka and Vilgalys 2013)

(Delph et al. 2002). Following a significant ANOVA, Tukey HSD (honest significant difference) post hoc tests were performed to further investigate which populations were significantly different. For $H$. bicruris, based on the available evidence for gene flow among populations (Magalhaes et al. 2011), so implying that individuals may not originate from where they were collected, we ran ANOVAs with population as a random factor. All analyses were done in R using packages "car" for the ANOVAs and "multcomp" for the Tukey HSD tests. Due to the small sample sizes in $H$ bricruris we tested the possibility of type II error (false negative for group differentiation) with $95 \%$ confidence by performing a power analysis using the R package "pwr".

Finally, to test whether developmental non-independence of the traits analysed might confound the interpretation of our results, we examined correlations between all the traits analysed, for each species separately but populations pooled together.

Correlation of geographic, phenotypic and genetic distances

We analysed how geographic and genetic distances are associated with phenotypic distances by correlating geographic and genetic matrices with phenotypic matrices based on population means of individual traits. Phenotypic distance matrices were obtained from population means for each individual trait, which were separately transformed into Euclidean distances. This was done for sexes pooled together and also males and females separately using SPSS 18.0 (SPSS Inc., Chicago IL, USA). The geographic distance matrix was estimated based on the latitude and longitude of each sampling location using GDMG v1.2.3 (http://biodiversityinformatics.amnh.org/open_source/gdmg/documentation.php). Genetic distance matrices $\left(\mathrm{F}_{\mathrm{ST}} / 1-\mathrm{F}_{\mathrm{ST}}\right)$ were obtained from Magalhaes et al. (2011). We tested the significance of relationships between matrices with partial Mantel tests based on 1,000 permutations as implemented in ARLEQUIN (Excoffier et al. 2005), where $X_{1}$ was 
the genetic matrix, $\mathrm{X}_{2}$ the geographic matrix and $\mathrm{Y}$ the phenotypic matrix. Additionally we also tested for an effect of latitude and longitude on trait size. In order to do this we converted geographic coordinates into decimal degrees and performed regressions of population means of each trait against latitude and longitude of their population, separately.

Correlation of phenotypic traits between plant and pollinator

We examined the correlations between individual traits potentially involved in plantpollinator/seed predator interactions by performing bivariate Pearson correlations between means and coefficients of variation of CTL, CTW and OvaL of $S$. latifolia populations and means and coefficients of variation of PrL, and OviL of H. bicruris populations. Correlations were calculated for each sex separately and for sexes pooled together. As moths need to reach the nectaries for feeding, which in female plants are located below the ovary, we also analysed the correlation between moth PrL and corolla tube length (estimated as sum of CTL and OvaL, see Fig. 1) of S. latifolia females.

\section{Results}

Phenotypic assignment of individuals to clusters

Analysing all populations and both sexes of S. latifolia together, Mclust identified two phenotypic clusters which captured most differences between male and female traits (BIC $=-3,775.014$, see Table S2 for details on BIC values for all numbers of clusters and models) (Fig. 2a). Not all individuals were accurately assigned to one of the clusters, with individuals in some populations being partly assigned to both clusters. Nonetheless, overall males were mostly assigned to cluster 1 and females to cluster 2 (Table S3a, Supporting Information). We estimated the error of the Mclust classification relative to the classification of plants by sex and obtained an error rate or 0.04 with 14 individuals misclassified by Mclust. A Chi square test confirmed that assignment of plants between the two clusters was significantly different from random $\left(\chi^{2}=55.747, d f=1, p<0.001\right)$, indicating sexual dimorphism in plant traits across the populations studied. When sexes were analysed separately, male plants were separated in two clusters (BIC $=-1,485.928)$ with 129 males (75\%) assigned to cluster 1 and $43(25 \%)$ to cluster 2 (see Table S2 for details on numbers of males form each populations assigned to each cluster). Out of the 43 males assigned to cluster 2, 34 (79 \%) belonged to Aarhus (15 males) and Kåseberga (19 males), populations where males had in general larger traits than those belonging to cluster 1 . Females on the other hand formed a single cluster when we performed the cluster analysis using all traits. However when we performed a cluster analysis based on ovary length alone, females were divided into two clusters (BIC $=-1,836.875)$ with females with an ovary length smaller than $8 \mathrm{~mm}$ belonging to cluster 1 and all others to cluster 2 . This meant that females from Sorby and Jonkoping and Stechendorf belonged to cluster 1.

Individuals of $H$. bicruris also formed two clusters (Fig. 2b) $(\mathrm{BIC}=-492.538)$. However, no signature of sexual dimorphism appeared here, as most individuals of both sexes were assigned to cluster 1 (Table S3b, Supporting Information). The error rate of Mclust compared to the known classification of moths by sex was 0.349 with 36 individuals misclassified by Mclust. The distribution of individuals among the two clusters was significantly different from random $\left(\chi^{2}=50.2, d f=1, p<0.001\right)$. 
(a) Silene latifolia

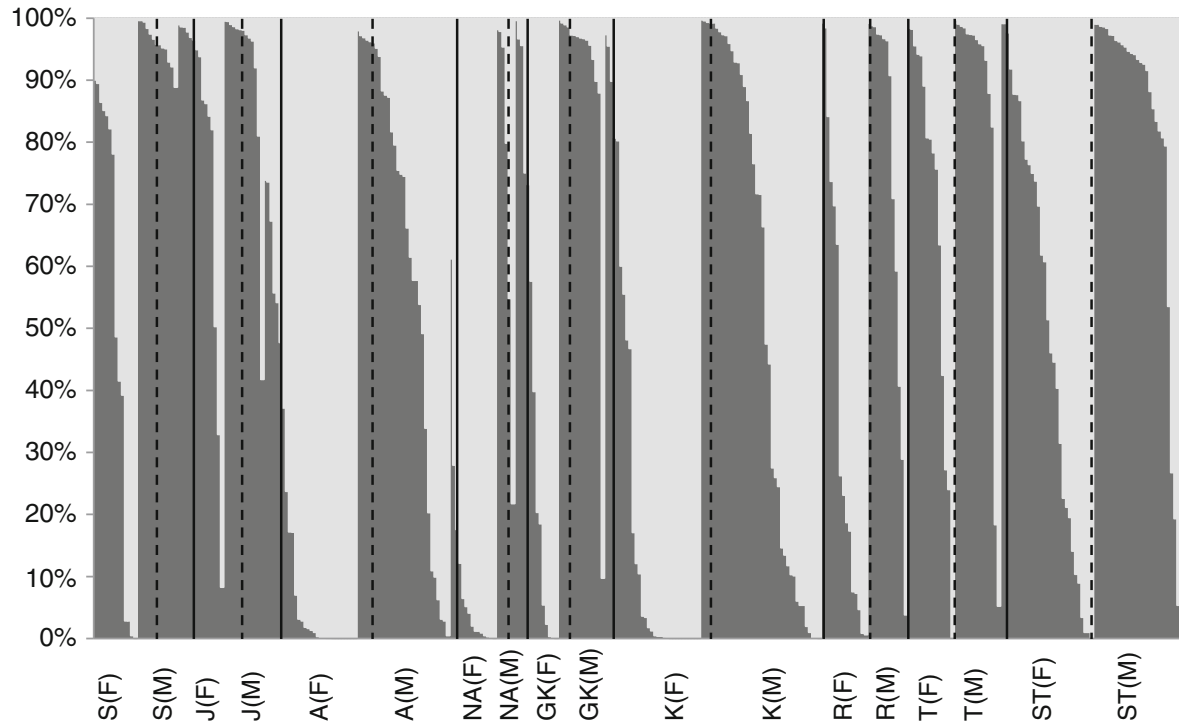

(b) Hadena bicruris

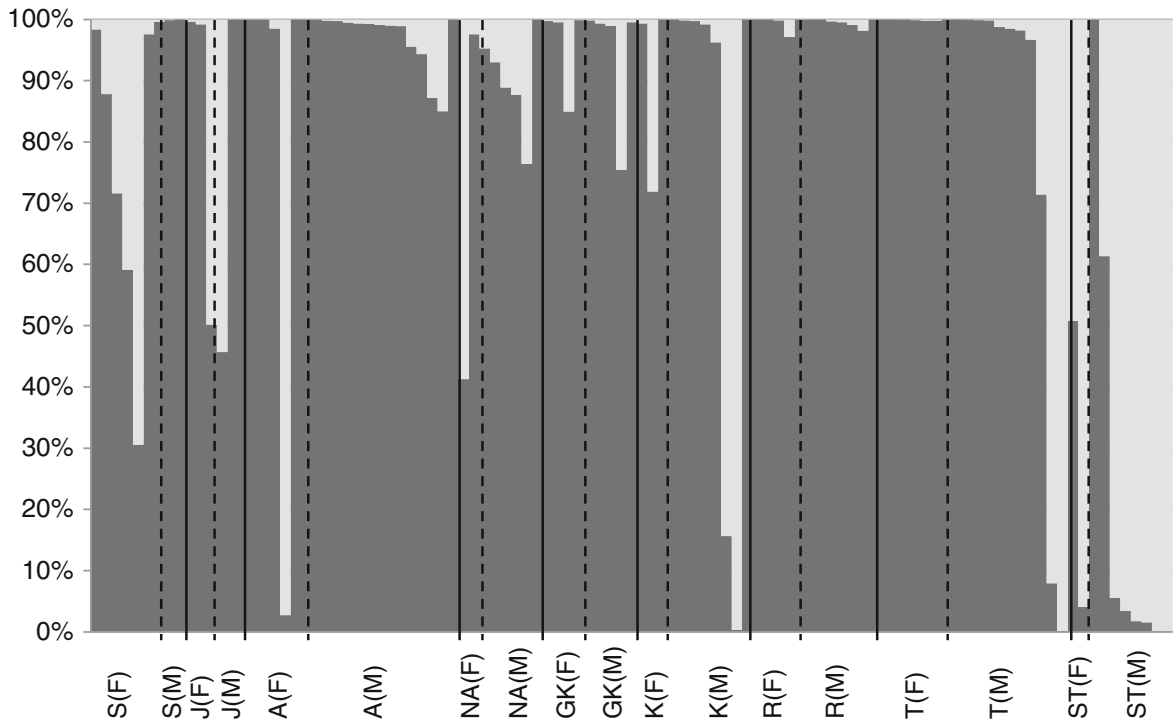

Fig. 2 Posterior probabilities of assignment of individuals to phenotypic clusters based on a CTW, CTL and PL for S. latifolia; and b PrL, WingA and ThW for H. bicruris. Each vertical line represents an individual and colours indicate the proportion of an individual's phenotype assigned to a particular cluster for the nine populations. Dashed lines separate populations and dotted lines separate sexes within populations 
Phenotypic variation and divergence between populations and sexes

\section{Silene latifolia}

All traits varied greatly among populations and sexes (see Table S1 for details on averages and standard deviations of traits per population and sex). We observed significant differences for all traits between populations and between sexes (MANOVA, Table S4 Supporting Information). When traits were analyzed separately, there were significant effects of sex, population and sex $x$ population interaction for all traits except PL, for which the interaction term was not significant (Table 1; Fig. S2a-c, Supporting Information, see also Table S5 for ANOVA coefficients). All traits were significantly different among males and females. Across all populations, males of S. latifolia were characterized by smaller corolla tube width, corolla tube length and petal length compared to females.

Not all traits were significantly different among all populations. Tukey HSD tests revealed that for corolla tube length 22 out of 36 pairwise comparisons between populations were significant with 7 of these comparisons being between Tolkamer and all other populations except Jonkoping, and 5 being between Jonkoping and other populations (see table S6 for detailed results on the Tukey HSD tests). For corolla tube width there were 9 significant pairwise comparisons, 7 of which involved Jonkoping and all other populations except Tolkamer, and 2 involving Tolkamer versus Aarhus and Norra Åsum. For petal length there were 8 significant pairwise comparisons, all of them involving the population Aarhus, which has on average the longest petal length. Among female plants there were significant differences in mean ovary length of different populations $\left(\mathrm{F}_{8,169}=5.045\right.$, $p<0.001$ ) (Table 1, Fig. S3d) with 4 pairwise comparisons among populations being significant, 3 of which involved the population with the shortest average ovary, Jonkoping.

Tukey HSD tests for the interaction sex*population revealed 107 out of 153 pairwise comparisons were significant, 80 of which were between males and females from different populations, 23 among females and 5 among males from different populations (see table S6 for detailed results on the Tukey HSD tests). For corolla tube width 54 out of 153 pairwise comparisons were significant, 38 of which were between different sexes from different populations, and 9 and 7 between females and between males from different populations respectively. Both traits were significantly different in 44 pairwise comparisons.

All traits were significantly positively correlated $(p<0.001)$ both when sexes were pooled and analysed separately (see Table S8 for detailed values on the correlation coefficients and $p$ values). This suggests these traits are not developmentally independent.

\section{Hadena bicruris}

Overall, morphological traits of $H$. bicruris differed significantly between populations and sex of the moth, but the interaction of the two was not significant (MANOVA, Supporting Information, Table S4). These results should be interpreted with some caution as power analyses showed that, given the small differences in averages between sexes in general and between sexes within each population, rejecting type II error (false negative for group differentiation) with $95 \%$ confidence would require higher sample sizes. The current sample size per population and sex would allow to detect with $95 \%$ confidence only a larger difference (effect size $>0.6$ ).

Following the MANOVA results we analysed only the main effects of population and sex for each trait separately. All traits were significantly different among populations (ANOVAs, Table 1, Table S5 for details on ANOVA coefficients). However, similar to 
Table 1 Results of type II SS ANOVAs for traits measured on males and females of S. latifolia and $H$. bicruris from nine populations across Northern/Central Europe

\begin{tabular}{|c|c|c|c|c|c|}
\hline Trait & Effect & $d f$ & Sum of squares & $F$ value & $\operatorname{Pr}(>F)$ \\
\hline \multicolumn{6}{|c|}{ Silene latifolia } \\
\hline \multirow[t]{4}{*}{ CTW } & Sex & 1 & 15.984 & 110.6973 & $<0.001 * * *$ \\
\hline & Pop & 8 & 8.723 & 7.5518 & $<0.001 * * *$ \\
\hline & Sex*Pop & 8 & 2.846 & 2.4641 & $0.01322 *$ \\
\hline & Residuals & 334 & 48.227 & & \\
\hline \multirow[t]{4}{*}{ CTL } & Sex & 1 & $7,225.6$ & $1,649.87$ & $<0.001 * * *$ \\
\hline & Pop & 8 & $1,051.4$ & 30.0101 & $<0.002 * * *$ \\
\hline & Sex*Pop & 8 & 248.8 & 7.1022 & $<0.003 * * *$ \\
\hline & Residuals & 354 & $1,550.3$ & & \\
\hline \multirow[t]{4}{*}{ PL } & Sex & 1 & 25.15 & 10.5709 & $0.00126 * *$ \\
\hline & Pop & 8 & 232.16 & 12.1971 & $<0.001 * * *$ \\
\hline & Sex*Pop & 8 & 26.96 & 1.4162 & 0.18816 \\
\hline & Residuals & 354 & 842.26 & & \\
\hline \multirow[t]{2}{*}{ OvaL } & Pop & 8 & 58.737 & 5.0449 & $<0.001 * * *$ \\
\hline & & 169 & 245.952 & & \\
\hline \multicolumn{6}{|c|}{ Hadena bicruris } \\
\hline \multirow[t]{3}{*}{ ThW } & Sex & 1 & 0.2316 & 2.9432 & 0.08943 \\
\hline & Pop & 8 & 2.4553 & 3.9011 & $0.0005 * * *$ \\
\hline & Residuals & 97 & 7.6315 & & \\
\hline \multirow[t]{3}{*}{ PrL } & Sex & 1 & 0.168 & 0.1122 & 0.73835 \\
\hline & Pop & 8 & $\mathbf{3 3 . 5 2 7}$ & 2.8052 & $0.00775 * *$ \\
\hline & Residuals & 96 & 143.42 & & \\
\hline \multirow[t]{3}{*}{ WingA } & Sex & 1 & 0.4121 & 4.652 & $0.03354 *$ \\
\hline & Pop & 8 & 3.7792 & 5.332 & $<0.001 * * *$ \\
\hline & Residuals & 95 & 8.416 & & \\
\hline \multirow[t]{2}{*}{ OviL } & Pop & 8 & 54.042 & 8.3982 & $<0.001 * * *$ \\
\hline & Residuals & 26 & 20.914 & & \\
\hline
\end{tabular}

Significant values in bold. For trait definition, see Methods and Fig. 2. Main Effects: Sex male or female plant/moth i.e. a significant main effect highlights sexual dimorphism in the trait. Pop population of origin

what happened to flower traits, not all populations were significantly different from each other. Tukey HSD tests revealed that 6 out of 36 pairwise comparisons were significant for thorax width, 4 of which involved the population Stechendorf and 2 the population Sorby (see Table S9 for detailed results on the Tukey HSD tests). The same number of pairwise comparisons was significant for wing area: 3 involved population Renkum and 3 involved population Stechendorf. The only pairwise comparison significant for Proboscis length was between populations Stechendorf and Arhus. Among populations of females, eight pairwise comparisons were significant, six of which involved the population with the lowest mean ovipositor length, Sorby. Aside from ovipositor length the population from Stechendorf was involved in the majority of the significant pairwise comparisons, which is a result of $H$. bicruris from this population having the lowest average values for all traits measured both when sexes were pooled together or analysed separately (Supporting 
Information; Fig. S2, Table S1). When population was considered a random factor, there were only significant differences for wing area among sexes $\left(\mathrm{F}_{1,102}=5.543, p=0.020\right)$.

All traits were significantly positively correlated when sexes were pooled $(p<0.05)$, suggesting they are not developmentally independent (see Table S8 for detailed values on the correlation coefficients and $p$ values). This was however not true when sexes were analysed separately, which could be a consequence of the low sample size.

Correlation between genetic, geographic and phenotypic distances

The partial Mantel tests revealed that for S. latifolia there was no significant correlation between any of the phenotypic matrices based on Euclidean distances and geographic or genetic distances (see Table S10 for details on results of Mantel tests' results). Additionally no traits changed significantly along the latitudinal and longitudinal gradient (see Table S11 for details on Pearson correlation coefficients and their $p$ values).

The partial Mantel tests revealed that for Hadena bicruris on the other hand there were a significant positive trend in the relationship between Euclidean distances based on proboscis length of males and geographic distances (Mantel test $\mathrm{r}=0.750, p=0.001$, $\mathrm{N}=9$ ) with geographic distances explaining $57 \%$ of the variation in proboscis length of H. bicruris males (Table S10, Supporting Information). Geographic distances also showed a positive relationship with wing area in females (Mantel test $(r)=0.436, p=0.013$, $\mathrm{N}=9$ ), males (Mantel test $\mathrm{r}=0.376, p=0.029$ ), and consequently also when sexes were pooled together (Mantel test $r=0.550, p=0.003$ ). Geographic distances between populations explained $12.7 \%$ of variation in wing area in females, $14.8 \%$ in males and $29.5 \%$ when sexes were pooled together. No significant correlations were observed between phenotypic and genetic distances.

Geographic position affected only male proboscis length, which increased significantly from more southern populations to more northern ones (Pearson correlation coefficient $(\mathrm{r})=0.896, p=0.001, \mathrm{~N}=9$ ) (Fig. 3). Consequently the same was true for when sexes were pooled together (Pearson correlation coefficient $(\mathrm{r})=0.748, p=0.020, \mathrm{~N}=9$ ) (see Table S11 for Pearson correlation coefficients for all traits and their $p$ values).

Correlations between phenotypic traits of plant and pollinator

Correlations between trait means for each population revealed a weak positive correlation between mean corolla tube length of $S$. latifolia and mean proboscis length of $H$. bicruris (Pearson correlation coefficient $(\mathrm{r})=0.673, p=0.047, \mathrm{~N}=9$ ) (Fig. 4a) (see Table S12 for details on correlation coefficients and $p$ values for all correlations between plant and moth traits). When moth sexes were analysed separately, this correlation only remained significant between mean corolla tube length and mean proboscis length of female $H$. bicruris (Pearson correlation coefficient $(\mathrm{r})=0.676, p=0.045, \mathrm{~N}=9$ ). Results were similar for the correlation between proboscis length and total corolla tube length, defined as the sum of ovary length and corolla tube length of female S. latifolia (this total corolla tube length accounts for the fact that nectaries are located below the ovary; Pearson correlation coefficient $(\mathrm{r})=0.677, p=0.045, \mathrm{~N}=9$ ), but mean proboscis length was always shorter than the total corolla tube length (Fig. 4b). The same was true for ovipositor length, which was always shorter than the the mean length of the corolla tube of the female flower of the same population, even though the correlation between the two was not significant (Pearson correlation coefficient $(r)=0.282, p=0.463$ ) (Fig. 4c). 


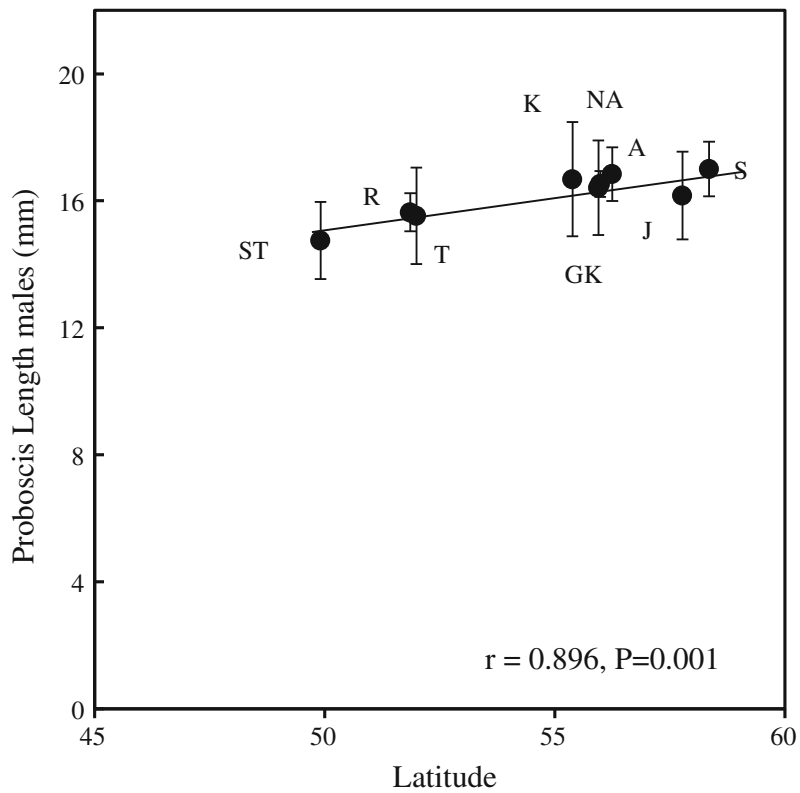

Fig. 3 Population mean ( \pm standard errors) for Proboscis Length in millimetres as a function of latitude. Proboscis tube length of $H$. bicruris males increased significantly with latitude. Pearson correlation coefficient $(r)$ its significance ( $p$ value) are also indicated

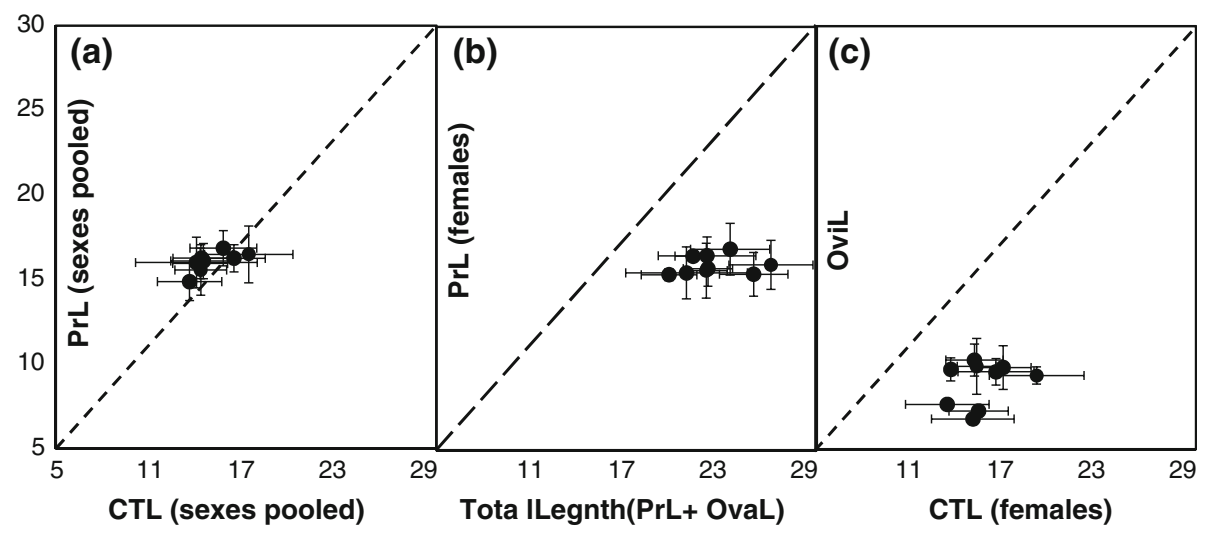

Fig. 4 Bivariate plots of means and standard deviations in millimetres of a Corolla tube length (CTL) of $S$. latifolia vs. proboscis length (PrL) of H. bicruris; b Total corolla tube length (corolla tube length + ovary length) vs. PrL of $H$. bicrurisfemales; and c CTL of $S$. latifolia females vs. ovipositor length (OviL) of $H$. bicruris females. The dashed lines are 1:1. lines. Pearson correlation coefficient ( $\mathrm{r}$ ) between traits and its significance ( $p$ value) are also indicated 


\section{Discussion}

The evolution of sexual dimorphism in plants and pollinators

We assessed traits assumed to play an important role for the interaction between the plant, $S$. latifolia and its pollinating seed predator, the moth $\mathrm{H}$. bicruris in multiple populations within a part of the native European range, where moth and plant co-occur, although this variation will reflect the combined effects of genetic and environmental sources of variation. As seed predation directly impacts female fitness, we expected selection on floral characters, especially those potentially linked to oviposition (e.g. Labouche and Bernasconi 2013), to have sex-specific consequences leading to sexual dimorphism. Consistent with this idea, male and female $S$. latifolia from the nine study populations constitute two phenotypically distinct clusters, where most females belong to one cluster and most males to another. Thus, sexual dimorphism seems to be consistent overall independently of population provenance. Extensive studies support the finding that sexual dimorphism, particularly in flower size, is very consistent in this species (Delph and Meagher 1995; Delph et al. 2002; Wright and Meagher 2004). This is most likely a consequence of conflicting selection on male and female flowers (Delph et al. 2004; Steven 2007) and future studies should explore further the contribution to selection on flower size due to interaction with $H$. bicruris. In a common garden experiment, longer female flowers, i.e. flowers with longer corolla tube than the moth ovipositor length, received more eggs outside the flower (on the petal), resulting in fewer successful fruit attacks compared to eggs placed inside the corolla tube (Labouche and Bernasconi 2013). Additionally, females with many, small flowers suffer higher abortion rates when attacked by $H$. bricruris than females with few, large flowers (Burkhardt et al. 2012).

Pressures from pollinators/seed predators and selection for increased fecundity and paternity are some of the most plausible explanations for phenotypic divergence between sexes, but they are not the only ones. Several populations of S. latifolia were infected with Microbotryum violaceum. Larger flowers, which are often females, are more likely to pick up spores from a contaminated insect (Elmqvist et al. 1993; Shykoff and Bucheli 1995; Bucheli and Shykoff 1999). Thus this could constitute an additional pressure on female flowers that could favour smaller female flowers and decrease differences between sizes of male and female flowers. In addition to all these different pressures, male and female floral characters can be genetically correlated and thus may not be able to respond independently to selection (Meagher 1994). In sum, S. latifolia faces a complex biotic system involving different pressures from pollinators/seed predators, selection for increased paternity and fecundity, and selection from pathogen fungi, all of which can jointly contribute to the evolution of flower size and floral sexual dimorphism.

Unlike $S$. latifolia, males and female $H$. bicruris did not diverge in any of the phenotypic traits analysed. This lack of strong sex-specific selection in the moth is consistent with a previous study that found no evidence for sex-specific visitation behavior, i.e. moths of either sex were equally likely to visit male and female flowers (Labouche and Bernasconi 2010). In our study of $H$. bicruris, differences between sexes seem to be restricted to the existence of an ovipositor to lay eggs inside the plants in females, but not in males. However more detailed analysis of other traits would be necessary to exclude sexual dimorphism on the whole phenotype as sexual dimorphism may be restricted to other traits, not included in this study, or become detectable only with larger sample sizes or decreased by the fact that some of the moths were reared under laboratory conditions and collected at different life stages. Also, we cannot rule out the possibility that the lack of sexual 
dimorphism in morphological traits is driven by intraspecific factors such as the determinants of moth mating success or constraints on flight ability that may act independently of the interaction with the plant.

Variation and divergence in the presence of gene flow

Geographically isolated populations did not constitute phenotypic clusters. Nonetheless individual ANOVAs found significant differences among populations for several traits of S. latifolia. Patterns of variation were different among traits and there seemed to be no consistent pattern of one population having the lowest values for all traits or the highest ones. This might explain why the cluster analyses failed to identify clusters corresponding to population provenance. The variation in the extent of differentiation and in the number of significantly different traits among populations and sexes suggests that both the strength and the target of selective pressures vary across populations, potentially reflecting variation in local ecological conditions, including the occurrence of co-pollinating species, presence/ absence of pathogens and patterns of gene flow. Consistent with this, a previous study on natural populations of $S$. latifolia found evidence for different selective pressures acting on plants from different sites and on different years and found that it is not always the same sex that is under higher selection across populations (Wright and Meagher 2004). Geographical distance per se does not appear to play a role in differentiation of any of the traits analysed. Nonetheless variation in corolla tube length was significantly correlated with moths' proboscis length, which along with other traits, is correlated with geographic distances. Hence, it is possible that there is an indirect effect of environmental distances on corolla tube length. Overall, our understanding of the patterns of variation and correlation involving corolla tube length, proboscis and ovipositor length may be increased in the future by studies that directly focus on the analysis of reciprocal selection of interaction traits and partition sources of variation that stem from abiotic environmental variation versus the plant-pollinator-seed predator interaction itself.

A different pattern appeared for the moth $H$. bicruris where variation in phenotypic traits was not only significantly different among populations, but in addition, both proboscis length in males and wing area in females were significantly correlated with geographic distances. This is an unexpected result as gene flow between these populations of $H$. bicruris appears to be high and mostly unrestricted (Magalhaes et al. 2011). Unfortunately we do not have sex-specific microsatellite markers for $H$. bicruris and we do not know whether dispersal distances are sex specific and whether the lack of an overall, significant genetic differentiation among moths from different populations is due to gene flow by both or one of the moth sexes. It is possible that extensive gene flow in one sex is masking the potential for differentiation in the other sex, but we would need both suitable markers and a larger sample size of both sexes to test this hypothesis. Alternatively, since trait variation in our field-collected individuals can result jointly from both genetic and environmental sources of variation, at least some of the observed correlations with geographic distances could reflect a geographic gradient on environmental effects on trait expression during development.

In sum, the significant differences among several traits in both S. latifolia and $H$. bicruris suggest selective pressures acting on one or both sexes in several of the populations. However, our results must be interpreted with some caution. These traits were measured on field collected individuals and thus variation in phenotype reflects also environmental and not only genetic variation. So we currently do not separate environmental plasticity from genetic based variation. Only measurements in a common garden 
setting could allow estimation of trait genetic variation, which could then be compared with neutral genetic variation (Pujol et al. 2008). Future studies comparing trait variation between field measurements and the measurements done on the same populations in the common garden for both species may be a first step in this direction. If trait differences are heritable we expect to find trait variation between populations and, if significantly higher than neutral genetic variation, would constitute strong evidence of selection acting on these traits.

Altogether these results suggest that geographic position and in some cases physical distances are important factors for variation of trait sizes in the moth, unlike levels of gene flow, which do not appear to be linked to phenotypic differences observed among populations (Magalhaes et al. 2011).

Correlation between plant and pollinator/seed predator traits: potential for coevolution?

Corolla tube length of $S$. latifolia correlated significantly, even if weakly, with $H$. bicruris proboscis length, suggesting that the two traits might have been under reciprocal selection (Janzen 1980) in at least in some of the populations (Thompson 2005). Similar relationships have been found in other groups of plant and pollinators (Anderson and Johnson 2008; Anderson et al. 2009). However, the value of such correlations as evidence for reciprocal selection and coevolution has been recently put into question (Nuismer et al. 2010). The main arguments are that reciprocal selection does not necessarily lead to significantly correlated traits (Thompson 1994) and that non-coevolutionary mechanisms could also explain correlations between traits of interacting species across sites (Janzen 1980; Nuismer et al. 2010). Hence, our findings are not sufficient to demonstrate reciprocal selection and coevolution in this system, nor to identify the forces generating this selection, which may be simultaneously due to the multiple aspects of this interaction, including predation (e.g. Labouche and Bernasconi 2013, corolla length), pollination and nectaring. Nevertheless, by pointing out the significant correlation between two traits involved in plant-pollinator interactions we aim at highlighting the potential that this system has for studies testing theories of coevolution, in particular since the interaction occurs across several species within these two genera (Kephart et al. 2006; Bernasconi et al. 2009; Castillo et al. 2013; Kula et al. 2013).

In conclusion, our analyses of variation in several traits potentially involved in this complex plant-insect interaction across nine European populations of S. latifolia and $H$. bricuris revealed consistent sexual dimorphism in the plant independent of population provenance, but not in the moth. Evolution of flower size and floral sexual dimorphism in $S$. latifolia is most likely a consequence of different pressures from pollinators/seed predators, selection for increased paternity and fecundity, and pathogen fungi. Several traits diverged among populations for both plant and moth, but a geographic gradient was only present for some traits in $H$. bricuris, which were correlated with geographic distances. Hence, while so far we have no direct evidence for coevolution in this system at the within species level, the replicated interactions of several species within these genera offer additional opportunities and strong potential for testing theories of coevolution. Also, phylogeographic, comparative and genetic studies would be instrumental to further our understanding of the complex interactions among these two organisms and the evolutionary forces that drive them.

Acknowledgments We thank Anne-Marie Labouche, David Nash, Nat Holland, Mario Vallejo-Marin and the referees for valuable discussion and for comments on the manuscript. We thank Gabriela Gleiser for the 
genetic data, Anne-Marie Labouche for the phenotypic data, Ladislaus Reser (Naturhistorisches Museum Luzern) for insect identification, Luca Scrucca for advice on the Mclust analyses, Dessislava SavovaBianchi for practical help in the laboratoy, Arjen Biere, Gösta Börjeson, Stefan Dötterl, Simon Laegaard, Honor Prentice, Per Toräng and Torbjörn Tyler for help and logistical support with field sampling, and Daniel Cherix for advice. This study was supported by the Swiss National Science Foundation (Grant No. 3100A0_122004/1 to GB).

\section{References}

Alexander HM (1989) An experimental field study of anther-smut disease of Silene alba caused by Ustilago violacea: genotypic variation and disease incidence. Evolution 43:835-847

Alexander HM, Antonovics J (1988) Disease spread and population dynamics of anther-smut infection of Silene alba caused by the fungus Ustilago violacea. J Ecol 76:91-104

Anderson B, Johnson SD (2008) The geographical mosaic of coevolution in a plant-pollinator mutualism. Evolution 62:220-225

Anderson B, Alexandersso R, Johnson SD (2009) Evolution and coexistence of pollination ecotypes in an African Gladiolus Iridaceae. Evolution 64:960-972

Anderson B, Terblanche JS, Ellis AG (2010) Predictable patterns of trait mismatches between interacting plants and insects. BMC Evol Biol 10:204

Anstett MC, Hossaert-McKey M, Kjellberg F (1997) Figs and fig-pollinators: coevolutionary conflicts in a coevolved mutualism. Trends Ecol Evol 12:94-99

Antonovics J, Alexander HM (1992) Epidemiology of anther-smut infection of Silene alba (=S. latifolia) caused by Ustilago violacea: patterns of spore deposition in experimental populations. Proc R Soc B 250:157-163

Avilés JM, Vikan JR, Fossøy F et al (2012) Egg phenotype matching by cuckoos in relation to discrimination by hosts and climatic conditions. Proc R Soc B 279:1471-2954

Bernasconi G, Antonovics J, Biere A et al (2009) Silene as a model system in ecology and evolution. Heredity 103:5-14

Biere A (2002) A plant pathogen reduces the enemy-free space of an insect herbivore on a shared host plant. Proc R Soc B 269:2197-2204

Bopp S, Gottsberger G (2004) Importance of Silene latifolia ssp alba and S. dioica Caryphyllacea as host plants of the parasitic pollinator Hadena bicrusis Lepidoptera, Noctuidae. Oikos 105:221-228

Bucheli E, Shykoff JA (1999) The influence of plant spacing on density-dependent versus frequencydependent spore transmission of the anther smut Microbotryum violaceum. Oecologia 119:55-62

Burkhardt A et al (2009) Benefits and costs to pollinating, seed-eating insects: the effect of flower size and fruit abortion on larval performance. Oecologia 161:87-98

Burkhardt A, Ridenhour BJ, Delph LF, Bernasconi G (2012) The contribution of a pollinating seed predator to selection on Silene latifolia females. J Evol Biol 25:461-472

Castillo DM, Kula AA, Fenster KA et al (2013) Specialist pollinating seed predator exhibits oviposition strategy consistent with optimal oviposition theory. Ecol Entomol 38:164-172

Cook JM, Rasplus JY (2003) Mutualists with attitude: coevolving fig wasps and figs. Trends Ecol Evol $18: 241-248$

Delph LF (2007) The genetic integration of sexually dimorphic traits in the dioecious plant, Silene latifolia. In: Fairbairn DJ, Blanckenhorn WU, Szekely T (eds) Sex, size and gender roles: evolutionary studies of sexual size dimorphism. Oxford University Press, New York, pp 115-123

Delph LF, Bell DL (2008) A test of the differential-plasticity hypothesis for variation in the degree of sexual dimorphism in Silene latifolia. Evol Ecol Res 10:61-75

Delph LF, Meagher TR (1995) Sexual dimorphism masks life-history trade-offs in the dioecious plant Silene latifolia. Ecology 76:775-785

Delph LF, Knapczyk FN, Taylor DR (2002) Among-population variation and correlations in sexually dimorphic traits of Silene latifolia. J Evol Biol 15:1011-1020

Delph LF, Gehring JL, Frey FM et al (2004) Genetic constraints on floral evolution in a sexually dimorphic plant revealed by artificial selection. Evolution 58:1936-1946

Delph LF, Arntz AM, Scotti-Saintagne C et al (2010) Quantitative trait loci and genomic architecture of sexual dimorphism in the dioecious plant Silene latifolia. Evolution 64:2873-2886

Dötterl S, Jürgens A (2005) Spatial fragrance patterns in flowers of Silene latifolia: lilac compounds as olfactory nectar guides? Plant Syst Evol 255:99-109 
Dufaÿ M, Anstett MC (2003) Confliscts between plants and pollinators that reproduce within influorescences: evolutionary variations on a theme. Oikos 100:3-14

Elmqvist T, Liu D, Carlsson U et al (1993) Anther-smut infection in Silene dioica: variation in floral morphology and patterns of spore deposition. Oikos 207-216

Elzinga JA, Bernasconi G (2009) Enhanced frugivory on invasive Silene latifolia in its native range due to increased oviposition. J Ecol 97:1010-1019

Elzinga JA, Biere A, Harvey JA (2002) The rearing of the gregarious koinobiont endoparasitoid Microplitis tristis Hymenoptera: braconidae on its natural host Hadena bicruris Lepidoptera: Noctuidae. Proc Sect Exp Appl Entomol Netherlands Entomol Soc 13:109-115

Elzinga JA, Turin H, van Damme JM et al (2005) Plant population size and isolation affect herbivory of Silene latifolia by the specialist herbivore Hadena bicruris and parasitism of the herbivore by parasitoids. Oecologia 144:416-426

Excoffier L, Estoup A, Cornuet JM (2005) Bayesian analysis of an admixture model with mutations and arbitrarily linked markers. Genetics 169:1727-1738

Fraley C, Raftery AE, Murphy TB, Scrucca L (2012) Mclust Version 4 for R: normal mixture modeling for model-based clustering, classification, and density estimation. Technical report no. 597, Department of Statistics, University of Washington

Golonka A, Vilgalys R (2013) Nectar inhabiting yeasts in Virginian populations of Silene latifolia (Caryophyllaceae) and co flowering species. Am Midl Nat 169:235-258

Goulson D, Jerrim K (1997) Maintenance of the species boundary between Silene dioica and S. latifolia red and white campion. Oikos 79:115-126

Hathaway L, Andersson S, Prentice HC (2009) Experimental crosses within European Silene latifolia Caryophyllaceae: intraspecific differentiation, distance effects, and sex ratio. Botany 87:231-240

Holland JN, DeAngelis DL, Schultz ST (2004) Evolutionary stability of mutualism: interspecific population regulation as an ESS. Proc R Soc B 271:1807-1814

Janzen DH (1980) When is it coevolution? Evolution 34:611-612

Jennersten O (1983) Butterfly visitors as vectors of Ustilago violacea spores between caryophyllaceous plants. Oikos 40:125-130

Jolivet C, Bernasconi G (2006) Experimental analysis of constitutive and induced defense in a plant-seedpredator system. Funct Ecol 20:966-972

Kephart S, Reynolds RJ, Rutter MT et al (2006) Pollination and seed predation on Silene and allied Caryophyllaceae: evaluating a model system to study the evolution of mutualisms. New Phytol 169:667-680

Kula AA, Dudash MR, Fenster CB (2013) Choices and consequences of oviposition by a pollinating seed predator, Hadena ectypa (Noctuidae), on its host plant, Silene stellata (Caryophyllaceae). Am J Bot 100:1148-1154

Labouche AM, Bernasconi G (2010) Male moths provide mutualistic benefits in the Silene latifolia-Hadena bicruris pollination, seed predation system. Funct Ecol 24:534-544

Labouche AM, Bernasconi G (2013) Cost limitation through constrained oviposition site in a plant-pollinator/seed predator mutualism. Funct Ecol 27:509-521

Magalhaes IS, Gleiser G, Labouche A et al (2011) Comparative population genetic structure in a plantpollinator/seed predator system. Mol Ecol 20:4618-4630

Meagher TR (1994) The quantitative genetics of sexual dimorphism in Silene latifolia (Caryophyllaceae). 2. Response to sex-specific selection. Evolution 48:939-951

Nuismer SL, Gomulkiewicz R, Ridenhour BJ (2010) When is correlation coevolution? Am Nat 175:525-537

Pellmyr O (2003) Yuccas, Yucca Moths, and coevolution: a review. Ann Missouri Bot Gard 90:35-55

Pettersson MW (1991) Flower herbivory and seed predation in Silene vulgaris Caryophyllaceae: effects of pollination and phenology. Holarct Ecol 14:45-50

Pujol B, Wilson AJ, Ross RIC et al (2008) Are QST-FST comparisons for natural populations meaningful? Mol Ecol 17:4782-4785

Richards CM, Emery SN, McCauley DE (2003) Genetic and demographic dynamics of small populations of Silene latifolia. Heredity 90:181-186

Roche BM, Alexander HM, Maltby AD (1995) Dispersal and disease gradients of anther-smut infection of Silene alba at different life stages. Ecology 76:1863-1871

Scheiner SM, Gurevitch J (2001) Design and analysis of ecological experiments. Oxford University Press, Oxford

Shykoff JA, Bucheli E (1995) Pollinator visitation patterns, floral rewards and the probability of transmission of Microbotryum violaceum, a venereal disease of plants. J Ecol 83:189-198

Steven JC (2007) Sexual dimorphism in the quantitative-genetic architecture of floral, leaf, and allocation traits in Silene latifolia. Evolution 61:42-57 
Thompson JN (1994) The coevolutionary process. Chicago University Press, Chicago

Thompson JN (2005) The geographic mosaic of coevolution. Chicago University Press, Chicago

Witt T, Jürgens A, Geyer R, Gottsberger G (1999) Nectar dynamics and sugar composition in flowers of Silene and Saponaria species Caryophyllaceae. Plant Biol 1:334-345

Wolfe LM (2002) Why alien invaders succeed: support for the escape-from-enemy hypothesis. Am Nat 160:705-711

Wright JW, Meagher TR (2004) Selection on floral characters in natural Spanish populations of Silene latifolia. J Evol Biol 17:382-395

Yu Q, Ellen ED, Wade MJ, Delph LF (2011) Genetic differences among populations in sexual dimorphism: evidence for selection on males in a dioecious plant. J Evol Biol 24:1120-1127 